\title{
To Assess Liraglutide's Therapeutic Effect in Patients with Type 2 Diabetes Mellitus Using Flash Glucose Monitoring System
}

\author{
Jianhong Yin ${ }^{1, *}$ \\ Minmin Han $\mathbb{D}^{1,2, *}$ \\ Linhui $\mathrm{Li}^{3}$ \\ Yang $\mathrm{Li}^{4}$ \\ Zi'ang Liu ${ }^{5,6}$ \\ Jing Yang ${ }^{\prime}$ \\ Yunfeng Liu'
}

'Department of Endocrinology, First Hospital of Shanxi Medical University, Taiyuan, Shanxi, People's Republic of China; ${ }^{2}$ First Clinical Medical College, Shanxi Medical University, Taiyuan, Shanxi, People's Republic of China; ${ }^{3}$ Department of Endocrinology, Taiyuan Central Hospital, Taiyuan, Shanxi, People's Republic of China; ${ }^{4}$ Department of Infectious Diseases, Linfen People's Hospital, Linfen, Shanxi, People's Republic of China; ${ }^{5}$ Third Clinical Medical College, Shanxi Medical University, Taiyuan, Shanxi, People's Republic of China; ${ }^{6}$ The Affiliated Bethune Hospital of Shanxi Medical University, Taiyuan, Shanxi, People's Republic of China

*These authors contributed equally to this work
Correspondence: Jing Yang; Yunfeng Liu Department of Endocrinology, First Hospital of Shanxi Medical University, No. 85 Jiefang Nan Road, Yingze District, Taiyuan, Shanxi Province, People's

Republic of China

Tel +86 I87034I6196

Fax +86 35I-4639758

Email yangjIm@I26.com; nectarliu@।63. com
Purpose: Liraglutide, a type of glucagon-like peptide-1 receptor agonist, has significant anti-hyperglycaemic activity without increasing the incidence of hypoglycaemia. In addition, it can improve $\beta$-cell function and insulin resistance. The flash glucose monitoring system (FGMS) is a novel method to document consecutive and detailed interstitial glucose levels, further reflecting blood glucose levels. This study aimed to investigate the therapeutic effect of liraglutide on blood glucose management (glucose variability, hyperglycaemia, and the incidence of hypoglycaemia), $\beta$-cell function, and insulin resistance in patients with diabetes. Patients and Methods: Thirty-three patients with type 2 diabetes mellitus were recruited in this study. On the basis of metformin monotherapy, these patients received liraglutide addon treatment for 3 months. The FGMS was used to document glucose levels before and after add-on treatment. Parameters of glucose variability, blood glucose levels at specific time periods, and the incidence of hypoglycaemia were assessed according to FGMS data and compared before and after liraglutide add-on treatment. Further, $\beta$-cell function and insulin resistance were assessed and compared before and after liraglutide add-on treatment.

Results: According to FGMS monitoring data, liraglutide add-on treatment significantly improved general, within-day, and day-to-day glucose variability and the glucose-target-rate. Further, the specifically analysed blood glucose levels at different time periods showed that blood glucose levels significantly decreased at nocturnal, fasting, and postprandial periods after add-on treatment. The incidence of hypoglycaemia was comparable during the whole day, daytime, and night-time according to the prespecified cutoffs $(3.9 \mathrm{mmol} / \mathrm{L}$ and $3.0 \mathrm{mmol} / \mathrm{L}$ ) before and after add-on treatment. Analysis of other assessed parameters revealed significant differences in glycosylated hemoglobin A1c and fasting blood glucose levels as well as parameters of $\beta$-cell function and insulin resistance before and after add-on treatment.

Conclusion: In type 2 diabetes mellitus, liraglutide treatment can effectively decrease glucose variability and ameliorate hyperglycaemia without increasing the incidence of hypoglycaemia. In addition, liraglutide can significantly improve the $\beta$-cell function and insulin resistance.

Keywords: glucagon-like peptide-1 receptor agonist, glucose variability, glucose target rate, time in range, $\beta$-cell function, insulin resistance

\section{Introduction}

Owing to its increasing prevalence and incidence, diabetes mellitus has always been a focal point of the general interest and a facing challenge worldwide. Due to its progressive nature, an optimum management should be individualised and modified 
along the course, in a complementary manner of maximum glycaemic effect and minimum adverse episodes. The commonly used antidiabetic agents such as insulin secretagogues or insulin are efficacious, but they confer a major risk of severe hypoglycaemia events. Liraglutide, ${ }^{1}$ a newly developed human glucagon-like peptide-1 (GLP-1) receptor agonist, contributed to hyperglycaemia amelioration in a strictly glucose-dependent manner without increasing the incidence of hypoglycaemia. A seminal review on a series of Phase III trials has provided strong evidence regarding the therapeutic effect of liraglutide as monotherapy or in combination with other oral antidiabetic drugs on blood glucose levels, $\beta$-cell function, incidence of hypoglycaemia, and weight loss. $^{2}$ Since then, a series of notable studies have provided data supporting its superiority in

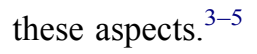

The flash glucose monitoring system (FGMS) is a new sensor-based scanning glucose monitoring system that indirectly reflects the blood glucose levels by detecting interstitial fluid glucose concentrations. It is convenient, visual, and easy to operate compared to the traditional self-monitoring blood glucose or continuous glucose monitoring system., Emerging data have confirmed the accuracy and safety of FGMS as well as its high patient acceptability and satisfaction. ${ }^{8-10}$ In this study, the FGMS was used for continuous glucose monitoring and providing blood glucose values throughout the day, allowing blood glucose control assessed from dynamic glucose monitoring perspective, and offering reliable and new insights into the therapeutic effect of liraglutide.

In this study, the FGMS was used to monitor the blood glucose alterations, including glucose variability (GV), blood glucose levels of specific periods, and the incidence of hypoglycaemic, in patients with type 2 diabetes mellitus (T2DM) before and after liraglutide add-on treatment, initiated due to poor glycemic control for $>2$ months on metformin monotherapy ( $1.5 \mathrm{~g}$ per day). Furthermore, $\beta$ cell function and insulin resistance (IR) were also assessed in these patients. In this study, we have provided realworld evidence and guidance for the clinical application of liraglutide in patients with T2DM.

\section{Patients and Methods}

The study was conducted at the First Hospital of Shanxi Medical University from August 2018 to April 2021. The study protocol was performed according to the Declaration of Helsinki and approved by the Ethics Committee of the
First Hospital of Shanxi Medical University (2018K008), and patients provided informed consent.

\section{Subjects}

Patients with T2DM (diagnosed according to the 1999 World Health Organization's Diabetes Diagnostic Criteria), who volunteered to receive liraglutide treatment for better blood glucose management, were enrolled in this study. The inclusion criteria were as follows: diabetes duration of 2-10 years; metformin ( $\geq 1.5$ g per day) monotherapy with poor glycaemic control; age between 18 and 70 years; baseline glycosylated hemoglobin A1c (HbA1c) level between $7 \%$ and $10 \%$; and body mass index $>24 \mathrm{~kg} /$ $\mathrm{m}^{2}$. Patients with diabetic nephropathy stage $\geq \mathrm{IV}$, those with severe liver and renal insufficiency, those with thyroid diseases or a family history of medullary thyroid carcinoma, and those with a history of pancreatitis and pancreatic tumours were excluded.

\section{Methods}

All the recruited patients received diabetes health education and wore the FGMS to monitor glucose levels for 2 weeks. After FGMS monitoring, these patients were treated with liraglutide for 3 months, during which the current hypoglycaemic regimen was continued (metformin: 1.5 g per day), and other GLP-1 receptor agonists and dipeptidyl peptidase-IV inhibitors could not be used. The recommended starting liraglutide dose was $0.6 \mathrm{mg}$ daily, which was adjusted every week in the first month until a final dose of $1.8 \mathrm{mg}$ per day. All patients were treated with liraglutide $1.8 \mathrm{mg}$ per day for the next 2 months. After the 3-month add-on treatment, the FGMS was used for 2 weeks to monitor blood glucose levels in these patients. Due to the inaccuracy of FGMS monitoring data at the first day of installation or other unexpected events, it was difficult to achieve a complete 2-week monitoring data. In total, 349-day and 364-day monitoring data were collected before and after add-on treatment, respectively. During the study period, all patients were requested to comply with a diabetic diet and exercise regimen. The meal was offered at 7:00, 12:00, and 18:00 during the day, consisting of $50 \%$ of carbohydrates, $35 \%$ of fats, and $15 \%$ of protein, with a caloric intake ratio of 2:4:4. A 30-minute exercise regimen (eg, walk, jogging, or biking) was recommended after a meal three to five times a week.

In addition, the levels of the following indices were tested before and after liraglutide add-on treatment: 
HbAlc, fasting blood glucose (FBG, mmol/L), and fasting insulin (FINS, $\mu \mathrm{IU} / \mathrm{mL}$ ). The IR and $\beta$-cell function were assessed using homeostasis model assessment (HOMA)IR, quantitative insulin sensitivity check index (QUICKI), fasting glucose-to-insulin ratio (G/I), and HOMA- $\beta$.

\section{Statistical Processing}

SPSS 19.0 and Sigmaplot 12.5 software were used for statistical analysis and graph construction. $\mathrm{P}<0.05$ was considered statistically significant. All values are expressed as mean \pm standard error of mean unless otherwise stated. Some data are expressed as median (first quartile, third quartile) due to the wide distribution.

The FGMS data were used to calculate the following parameters: 24-hour mean of blood glucose (24hMBG) and coefficient variable $(\mathrm{CV})$ representing general $\mathrm{GV}$; standard deviation of blood glucose (SDBG), mean amplitude of glycaemic excursions (MAGE) and large amplitude of glycaemic excursions (LAGE) indicating withinday $\mathrm{GV}$; mean of daily differences (MODD), area of interquartile range (IQR), and area of interdecile range (IDR) showing day-to-day GV; and percentile time (PT, PT1: blood glucose level <3.9 mmol/L; PT2: 3.9-10 $\mathrm{mmol} / \mathrm{L}$; and PT3: $>10 \mathrm{mmol} / \mathrm{L}$ ), time in range (TIR), and time out of range (TOR) representing the glucosetarget-rate. These parameters were compared before and after add-on treatment using a $t$-test (for normally distributed data) or the Mann-Whitney test (for skewed data). The normal range of the blood glucose levels was set as 3.9-10.0 mmol/L.

The blood glucose levels were specifically analysed at five periods before and after add-on treatment, including nocturnal period (0:00-6:00), fasting period (6:00-7:00), and postprandial periods (7:00-9:00, 12:00-14:00, and 18:00-20:00). The mean blood glucose level at each time point in each patient were drawn as multiple-line and scatter diagram and the area under the curve (AUC) of each analysed period was calculated. A $t$-test or the MannWhitney test was used to compare the AUC in each time period before and after add-on treatment.

The incidence of hypoglycaemia was evaluated during three time periods, including the whole day (0:00-24:00), daytime (6:00-24:00), and night-time (0:00-6:00). A blood glucose $<3.9 \mathrm{mmol} / \mathrm{L}$ or $3.0 \mathrm{mmol} / \mathrm{L}$ were used as the cut-off. A $t$-test or the Mann-Whitney test was used to compare the incidence of hypoglycaemia during the time periods before and after add-on treatment.
The parameters of IR and $\beta$-cell function were calculated using the following formulas: HOMA-IR = $(\mathrm{FBG} \times \mathrm{FINS}) / 22.5 ;$ QUICKI $=1 /[(\operatorname{logFINS})+(\operatorname{logFBG})]$; $\mathrm{G} / \mathrm{I}=\mathrm{FBG} / \mathrm{FINS}$; and HOMA- $\beta=(20 \times \mathrm{FINS}) /(\mathrm{FBG}-$ 3.5). These indices, $\mathrm{HbA} 1 \mathrm{c}, \mathrm{FBG}$, and FINS were compared before and after add-on treatment using a $t$-test or the Mann-Whitney test.

\section{Results \\ General Characteristics of the Recruited Patients}

The clinical characteristics of the patients are showed in Table 1. A total of 33 patients (18 males and 15 females) aged $(50.061 \pm 0.774)$ years with a duration of diabetes mellitus of $6.455 \pm 0.329$ years were enrolled.

\section{Glucose Variability}

The parameters representing general GV showed that 24hMBG was significantly decreased after add-on treatment compared to that before $(\mathrm{P}<0.001)$, while $\mathrm{CV}$ was comparable before and after add-on treatment (Figure 1). Parameters of within-day GV indicated significantly lower SDBG and LAGE after add-on treatment than that before ( $\mathrm{P}<0.001$, and $\mathrm{P}=0.002$, respectively); however, no significant difference was found in MAGE before and after treatment (Figure 2). Regarding day-to-day GV, significant differences were identified in MODD $(\mathrm{P}=0.002)$, and areas of IQR $(\mathrm{P}<0.001)$ and IDR $(\mathrm{P}=0.001)$ before and after add-on treatment (Figure 2). Indices representing the glucose-target-rate revealed significantly decreased PT3 and TOR and significantly increased PT2 and TIR after add-on treatment compared to that before. However, PT1 was not significantly different before and after add-on treatment, implying that liraglutide treatment satisfied the antidiabetic effect without increasing the incidence of hypoglycaemia (Figure 1).

\section{Blood Glucose Levels at Different Time Periods}

According to Figure 3A, there was a great tendency of reduced general blood glucose level after add-on treatment compared to that before. Further comparison of the blood glucose levels at specific periods before and after add-on treatment showed significantly decreased blood glucose levels during nocturnal, fasting, and postprandial periods (Figure 3). 
Table I General Characteristics of the Recruited Subjects

\begin{tabular}{|l|c|}
\hline Parameters & Values \\
\hline Gender (male:female) & $18: 15$ \\
Age (years) & $50.061 \pm 0.774$ \\
Duration of diabetes (years) & $6.455 \pm 0.329$ \\
Body mass index $\left(\mathrm{kg} / \mathrm{m}^{2}\right)$ & $27.509 \pm 0.759$ \\
Metformin dose $(\mathrm{g} /$ day) & 1.5 \\
\hline
\end{tabular}

Note: Part of the data were denoted as mean \pm standard error of mean.

\section{Hypoglycaemia Incidence}

There was no marked difference revealed in the incidence of hypoglycaemia episodes during the whole day, daytime, and night-time according to the prespecified cutoffs (3.9 $\mathrm{mmol} / \mathrm{L}$ and $3.0 \mathrm{mmol} / \mathrm{L}$ ) before and after add-on treatment (Table 2).

\section{Metabolic Indicators}

The results of the metabolic indicators are showed in Table 3. There was no significant difference in body mass index before and after treatment. A significant difference was identified in the parameters of $\mathrm{HbA} 1 \mathrm{c}$, and FBG before and after add-on treatment $(\mathrm{P}<0.001$, and $\mathrm{P}=$ 0.003 , respectively). The parameters of IR revealed that significantly decreased HOMA-IR $(P=0.003)$ and $G / I(P$ $=0.012)$, and increased QUICKI $(\mathrm{P}=0.024)$ after add-on treatment compared to that before. Comparison of HOMA$\beta$ before and after add-on treatment indicated a significant difference $(\mathrm{P}<0.001)$. No significance was found in FINS levels before and after treatment. Taken together, these results demonstrated significantly improved general blood glucose levels, IR, and $\beta$-cell function.

\section{Discussion}

Diabetes mellitus is a complex entity, characterized by IR and progressive $\beta$-cell deterioration, manifested as hyperglycaemia state, usually accompanied with a constellation of complications. There is overwhelming evidence for the involvement of IR and hyperglycaemia in the pathogenesis
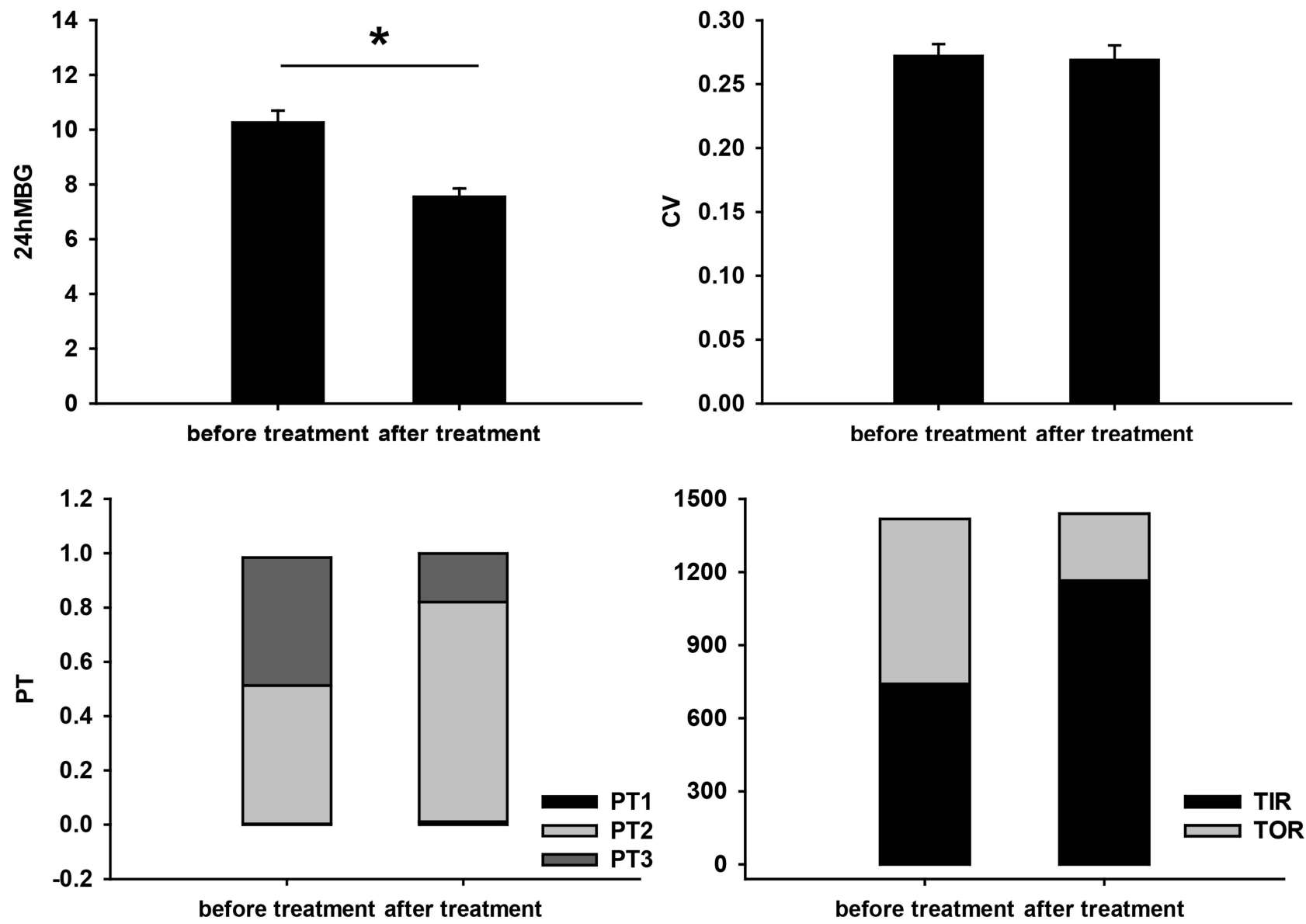

Figure I Comparison of general GV and the glucose-target-rate before and after liraglutide add-on treatment. The values are presented as mean $\pm \mathrm{SEM}$. $*$ Represents significant difference $(P<0.05)$.

Abbreviations: GV, glucose variability; SEM, standard error of mean. 

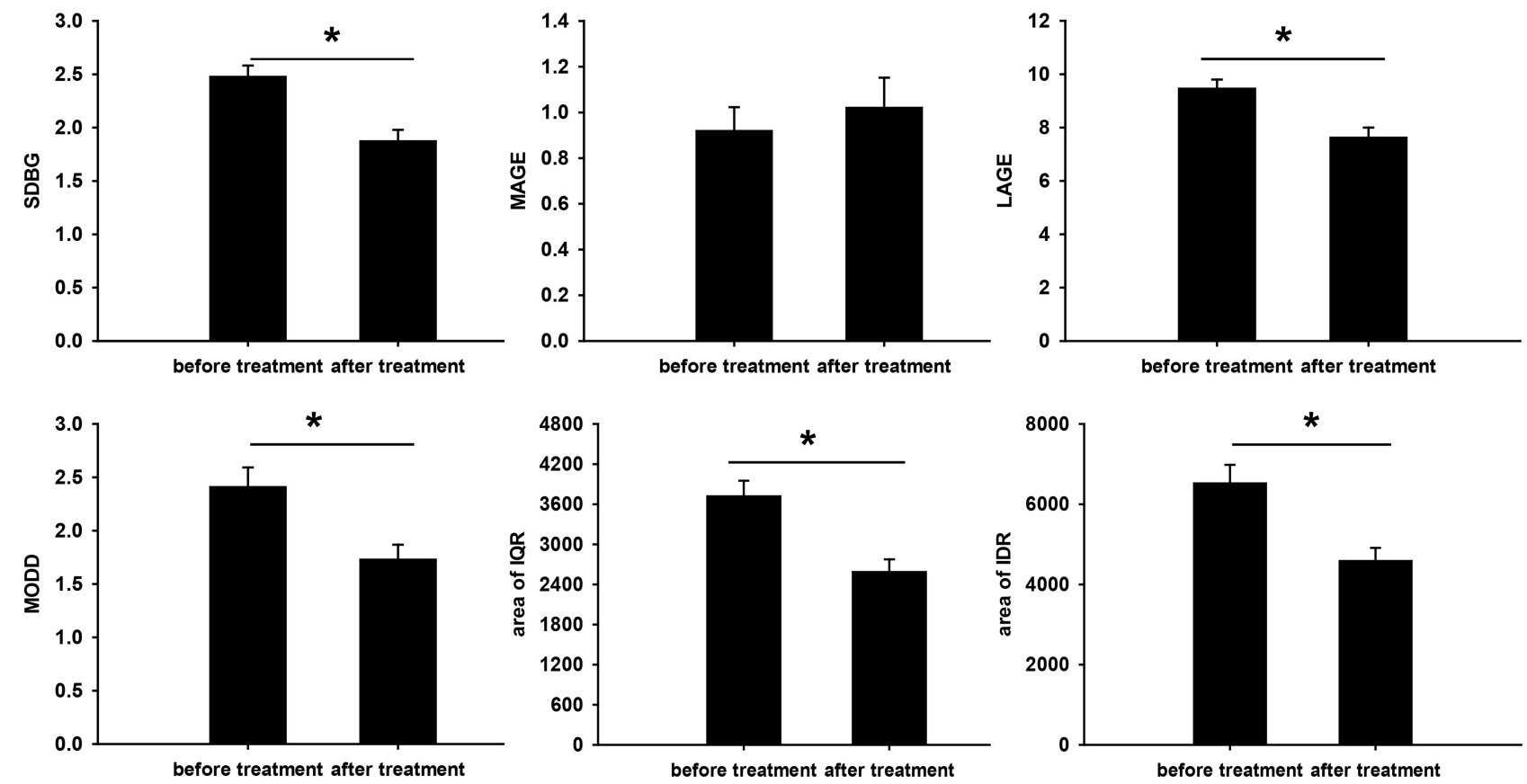

Figure 2 Comparison of within-day and day-to-day GV before and after liraglutide add-on treatment. The values are presented as mean \pm SEM. *Represents significant difference $(\mathrm{P}<0.05)$

Abbreviations: GV, glucose variability; SEM, standard error of mean.

of vascular endothelial injury, inflammation reaction, and oxidative stress, and ensuing micro- and macro-angiopathy in patients with diabetes. ${ }^{11}$ Another perspective that should be underlined was the valued role of GV in this setting, which is an emerging field that is attracting the interests of many researchers. ${ }^{12,13}$ Reducing GV, namely the amplitude, frequency, and duration of the upward and downward glucose fluctuations, is a key component of the glucoselowering therapy. Over the past few years, a considerable body of evidence from studies has indicated improved prognosis of diabetes-related complications when to intervene in GV and maintain a stable glycaemic state. ${ }^{14}$ In addition, some proof has suggested the major and indispensable role of hypoglycaemia in the progression of diabetes and its complications. ${ }^{15}$ Therefore, in patients with diabetes, simply controlling of blood glucose within the normal range may have a preventive effect on disease progression, but it is clinically crucial to pay attention to other contributors, in particular GV and occurrence of hypoglycemia.

Liraglutide, a novel GLP-1 receptor agonist, has fuelled considerable interests worldwide due to its safe and effective hypoglycaemic effect, and various clinical benefits. Large amounts of researches have provided ground for its therapeutic effects. ${ }^{2-5}$ Recently, the dual GLP-1/glucagon receptor agonists, SAR425899, sparked much interest in the scientific community. Comparison investigation studies were performed between liraglutide and SAR425899, showing comparable blood glucose improvement and higher enhancement of $\beta$-cell function with SAR425899 in patients with obesity and T2DM. ${ }^{16}$ In addition, clinical studies comparing liraglutide with other GLP-1 receptor agonists have reported inconsistent results. ${ }^{5,17,18}$ Therefore, this study explored the liraglutide's therapeutic effect from a continuous glucose monitoring perspective, promising to offer real-world credence for its widespread application.

Analysis of GV parameters revealed that liraglutide add-on therapy decreased the general, within-day, and dayto-day GV, and improved the glucose target rate. Our findings are consistent with those of previous researches and further expanded and added credence to previous findings in this area. In the past few years, ever-growing researches demonstrated the decreased GV with liraglutide treatment, focusing on the indices of MAGE, LAGE, MODD, and SD. ${ }^{14}$ This study provided novel evidence on general, within-day, and day-to-day GV, and the glucose-target-rate as a proper reference for clinical practice.

To date, the majority of evidence collected suggests the involvement of fluctuating blood glucose levels in the pathogenesis of diabetic complications, via increased production of reactive oxygen species and 

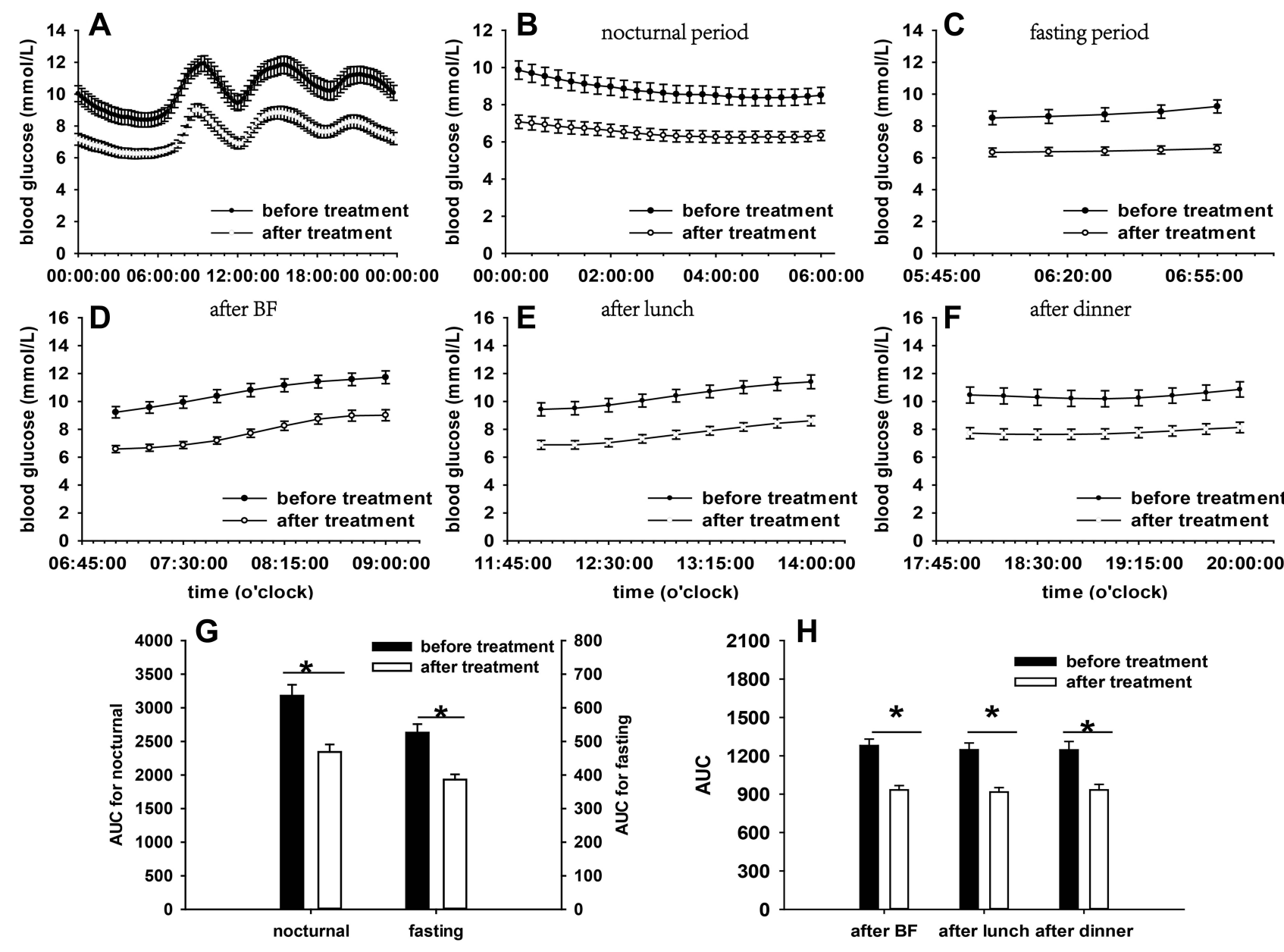

Figure 3 Comparisons of mean blood glucose levels and AUCs before and after liraglutide add-on treatment. Mean blood glucose levels: (A) during the day; (B) at nocturnal period; (C) at fasting period; (D) after BF; (E) after lunch; (F) after dinner. AUC: (G) at nocturnal and fasting periods; (H) at postprandial periods. The values are presented as mean \pm SEM. *Represents statistically different AUC in this time period $(P<0.05)$.

Abbreviations: AUC, area under curve; BF, breakfast; SEM, standard error of mean.

advanced glycation end products, for example. ${ }^{19,20}$ Furthermore, studies investigating the relationship between TIR and diabetic macro- or micro-angiopathy have highlighted negative correlations. ${ }^{21-24}$ The increment of TIR is proportional to vascular lesion occurrence, such as, retinopathy and microalbuminuria, and mortality hazard reduction. This study observed significantly decreased GV, specifically, the TIR increased by $57 \%$ after liraglutide treatment compared to that before. Therefore, liraglutide treatment improved $\mathrm{GV}$ in the recruited patients, which further decreased the risks of diabetic complications along the course of the disease. Long-term observational studies are required to prove this conclusion.

Table 2 The Incidence of Hypoglycaemia Before and After Treatment

\begin{tabular}{|l|l|l|l|l|l|}
\hline & & & Before Treatment & After Treatment & P value \\
\hline Hypoglycaemia incidence (\%) & Hypoglycaemia cutoff: $3.9 \mathrm{mmol} / \mathrm{L}$ & $\begin{array}{l}\text { The whole day } \\
\text { Daytime } \\
\text { Night-time }\end{array}$ & $\begin{array}{l}0(0,0) \\
0(0,0) \\
0(0,0)\end{array}$ & $\begin{array}{l}0(0,1.09) \\
0(0,0.537) \\
0(0,0.272)\end{array}$ & $\begin{array}{l}0.123 \\
0.076 \\
0.12\end{array}$ \\
\hline Hypoglycaemia incidence (\%) & Hypoglycaemia cutoff: $3.0 \mathrm{mmol} / \mathrm{L}$ & $\begin{array}{l}\text { The whole day } \\
\text { Daytime }\end{array}$ & $\begin{array}{l}0(0,0) \\
0(0,0)\end{array}$ & $\begin{array}{l}0(0,0) \\
0(0,0) \\
0(0,0)\end{array}$ & $\begin{array}{l}0.24 \\
0.988 \\
0.189\end{array}$ \\
\hline
\end{tabular}

Notes: The data were shown as median (first quartile, third quartile). The whole day (0:00-24:00), daytime (6:00-24:00), and night-time (0:00-6:00). 
Table 3 Comparison of Metabolic Indicators Before and After Treatment

\begin{tabular}{|l|c|c|c|}
\hline Variables & Before Treatment & After Treatment & P value \\
\hline Body mass index $\left(\mathrm{kg} / \mathrm{m}^{2}\right)$ & $27.509 \pm 0.759$ & $26.893 \pm 0.784$ & 0.261 \\
HbAIc $(\%)$ & $7.876 \pm 0.21 \mathrm{I}$ & $6.221 \pm 0.235$ & 0.001 \\
FBG $(\mathrm{mmol} / \mathrm{L})$ & $9.821 \pm 0.331$ & $6.289 \pm 0.198$ & 0.003 \\
FINS $(\mu \mathrm{lU} / \mathrm{mL})$ & $17.942 \pm 1.496$ & $18.426 \pm 1.847$ & 0.934 \\
HOMA-IR & $8.016 \pm 0.759$ & $5.344 \pm 0.569$ & 0.003 \\
QUICKI & $0.472 \pm 0.0137$ & $0.522 \pm 0.0179$ & 0.024 \\
G/I & $0.771 \pm 0.0926$ & $0.469 \pm 0.0447$ & 0.012 \\
HOMA- $\beta$ & $58.847 \pm 5.35$ & $136.757 \pm 13.317$ & 0.001 \\
\hline
\end{tabular}

Note: The data were denoted as mean \pm standard error of mean.

Abbreviations: HbAlc, glycosylated hemoglobin Alc; FBG, fasting blood glucose; FINS, fasting insulin; HOMA-IR, homeostasis model assessment-insulin resistance; QUICKI, quantitative insulin sensitivity check index; G/l, fasting glucose-to-insulin ratio.

Blood glucose levels during nocturnal, fasting, and postprandial periods significantly decreased after add-on treatment compared to those before. In addition, the incidence of hypoglycaemia was comparable during the whole day, daytime, and night-time with both the cutoffs before and after add-on treatment. These results indicate that liraglutide treatment in patients with can produce a positive therapeutic effect in diabetic management without increasing the incidence of hypoglycemia. These results add to the existing body of evidence on the effectiveness of liraglutide in the glycaemic control and its safety. The landmark Phase III clinical trials reported significantly decreased fasting and postprandial blood glucose levels without an increased risk of hypoglycaemia. ${ }^{25-27}$ Consistent with the previously established conclusion, our study provided convincing proof at time periods perspective, showing significantly decreased blood glucose levels on the whole, and at nocturnal, fasting, and postprandial periods.

It is well established that liraglutide, a GLP-1 receptor agonist, is akin to human endogenous GLP-1 with $97 \%$ structure homology, exhibiting similar pharmacodynamics effects, longer half-life, and refractory to degradation compared to that of human GLP-1. ${ }^{1}$ Classified as incretin mimetics, liraglutide promotes glucose-dependent insulin secretion and suppresses glucagon secretion, leading to postprandial glycaemic control. Further, the effect of liraglutide on gastric emptying and satiety also plays a critical role in decreasing postprandial blood glucose levels, except for the weight-loss effect. In addition, reduced hepatic glucose output may be relevant to the fasting glycaemic control. ${ }^{28,29}$

Data on the other metabolic parameters indicated significant differences in HbAlc level, FBG level, HOMA-
IR, QUICKI, G/I, and HOMA- $\beta$ before and after add-on treatment. According to these results, one can speculate that liraglutide treatment significantly improved glycaemic control, IR, and $\beta$-cell function. However, no significance was found in body mass index before and after treatment. This study did not observe a significant weight loss effect, which is inconsistent with the findings of previous research. ${ }^{4}$ Long-term follow-up for weight monitoring was arranged to evaluate the weight loss efficacy of liraglutide.

Consistent with the results of this study, several converging lines of data have suggested the superiority of liraglutide in terms of $\beta$-cell preservations. The underlying evidence is based on observations of liraglutide's protective effect in $\beta$-cell along with suppressing effect in their apoptosis. ${ }^{30,31}$ Previous studies have struggled to prove the insulin sensitivity restoration with liraglutide intervention in a diabetic animal model, illuminating the involvement of reversely increased gene expression of glucose transporter 4 and counteraction of endoplasmic reticulum stress. ${ }^{32,33}$ However, clinical studies evaluating the effect of liraglutide treatment on IR in the Asian population have yielded to negative outcomes. ${ }^{34,35}$ In the current study, significantly improved IR was noted after liraglutide addon treatment, lending support to the established conclusion in the animal experiments. Notably, further studies will be necessary to elucidate the therapeutic effect of liraglutide on IR with large samples.

In summary, the management of patients with T2DM with poor glycaemic control is a long and arduous journey, among which lifestyle adaptation is acknowledged as a key component. On the basis of diet and exercise modification, the use of liraglutide combined with oral hypoglycaemic therapy can achieve early and long-term protection of islet 
$\beta$ cells and improve IR, effectively manage GV and hyperglycaemia without increasing the incidence of hypoglycaemia, further suspending the development of diabetic complications. As a new clinical drug, liraglutide's efficacy has been proven by previous reliable studies. The results of this study illustrated its therapeutic effect from a dynamic blood glucose monitoring perspective. However, it is expected that the larger sample sizes and even multi-center clinical observations will verify our results in the future.

\section{Conclusion}

This study showed the therapeutic effect of liraglutide from a dynamic glucose monitoring perspective. Using FGMS, this study demonstrated that liraglutide add-on treatment achieved desired outcomes in terms of GV, period glucose levels, and incidence of hypoglycemia. In addition, $\beta$-cell function and IR also significantly improved after treatment. Notably, large-scale or multicentre researches are needed to confirm these results. Owing to the ever-growing attention on the adverse effect of GV, it is of paramount importance to exploit FGMS to monitor glucose levels in T2DM to make medication adjustments to reduce GV and increase TIR, further mitigating the progression of diabetic complications.

\section{Abbreviations}

GLP-1, glucagon-like peptide-1; FGMS, flash glucose monitoring system; T2DM, type 2 diabetes mellitus; GV, glucose variability; IR, insulin resistance; FBG, fasting blood glucose; HbA1C, glycosylated hemoglobin A1c; FINS, fasting insulin; HOMA, homeostasis model assessment; QUICKI, quantitative insulin sensitivity check index; G/I, fasting glucose-to-insulin ratio; 24hMBG, 24hour mean blood glucose; $\mathrm{CV}$, coefficient variable; SDBG, standard deviation of blood glucose; MAGE, mean amplitude of glycaemic excursions; LAGE, large amplitude of glycaemic excursions; MODD, mean of daily differences; $\mathrm{IQR}$, interquartile range; IDR, interdecile range; PT, percentile time; TIR, time in range; TOR, time out of range.

\section{Consent for Publication}

All authors provided written consent to publish this study.

\section{Acknowledgments}

The coauthors thank the National Natural Science Foundation of China (81770776, 81973378, 82073909), Research Project Supported by Shanxi Scholarship Council of China (2020-0172), and Natural Science
Foundation of Shanxi Province (201901D111353) for their support.

\section{Author Contributions}

All authors contributed to data analysis, and drafting or revising the article, have agreed on the journal to which the article will be submitted, gave final approval of the version to be published, and agree to be accountable for all aspects of the work.

\section{Disclosure}

The authors report no conflicts of interest in this work.

\section{References}

1. Ram K, Kamlesh K, Melanie JD. Liraglutide for type 2 diabetes mellitus. Expert Opin Biol Ther. 2011;7:951-959.

2. Blonde L, Russell-Jones D. The safety and efficacy of liraglutide with or without oral antidiabetic drug therapy in type 2 diabetes: an overview of the LEAD 1-5 studies. Diabetes Obes Metab. 2009;11(Suppl 3):26-34. doi:10.1111/j.1463-1326.2009.01075.x

3. Tanaka K, Saisho Y, Manesso E, et al. Effects of liraglutide monotherapy on beta cell function and pancreatic enzymes compared with metformin in Japanese overweight/obese patients with type 2 diabetes mellitus: a subpopulation analysis of the KIND-LM randomized trial. Clin Drug Investig. 2015;35(10):675-684. doi:10.1007/s40261-0150331-5

4. Davies MJ, Bergenstal R, Bode B, et al. Efficacy of liraglutide for weight loss among patients with type 2 diabetes: the SCALE diabetes randomized clinical trial. JAMA. 2015;314(7):687-699. doi:10.1001/ jama.2015.9676

5. Kapitza C, Forst T, Coester HV, et al. Pharmacodynamic characteristics of lixisenatide once daily versus liraglutide once daily in patients with type 2 diabetes insufficiently controlled on metformin. Diabetes Obes Metab. 2013;15(7):642-649. doi:10.1111/dom.12076

6. Rai S, Hulse A, Kumar P. Feasibility and acceptability of ambulatory glucose profile in children with type 1 diabetes mellitus: a pilot study. Indian J Endocrinol Metab. 2016;20(6):790-794. doi:10.4103/22308210.192894

7. Garg SK, Akturk HK. Flash glucose monitoring: the future is here. Diabetes Technol Ther. 2017;19(S2):S1-S3.

8. Linong J, Xiaohui G, Lixin G, et al. A multicenter evaluation of the performance and usability of a novel glucose monitoring system in Chinese adults with diabetes. J Diabetes Sci Technol. 2017;11 (2):290-295. doi:10.1177/1932296816662884

9. Bailey T, Bode BW, Christiansen MP, et al. The performance and usability of a factory-calibrated flash glucose monitoring system. Diabetes Technol Ther. 2015;17(11):787-794. doi:10.1089/ dia. 2014.0378

10. Sato T, Oshima H, Nakata K, et al. Accuracy of flash glucose monitoring in insulin treated patients with type 2 diabetes. J Diabetes Investig. 2019;10(3):846-850. doi:10.1111/jdi.12954

11. Stratton IM, Adler AI, Neil HA, et al. Association of glycaemia with macrovascular and microvascular complications of type 2 diabetes (UKPDS 35): prospective observational study. BMJ. 2000;321 (7258):405-412. doi:10.1136/bmj.321.7258.405

12. Lachin JM, Genuth S, Nathan DM, et al. Effect of glycemic exposure on the risk of microvascular complications in the diabetes control and complications trial revisited. Diabetes. 2008;57(4):995-1001. doi: $10.2337 / \mathrm{db} 07-1618$ 
13. Gerbaud E, Darier R, Montaudon M, et al. Glycemic variability is a powerful independent predictive factor of midterm major adverse cardiac events in patients with diabetes with acute coronary syndrome. Diabetes Care. 2019;42(4):674-681. doi:10.2337/dc18-2047

14. Xing Y, Chen J, Zhao L, et al. Analysis of the effect of liraglutide on glycemic variability in patients with type 2 diabetes. Endocr $J$. 2020;67(4):455-468. doi:10.1507/endocrj.EJ19-0530

15. Bedenis R, Price AH, Robertson CM, et al. Association between severe hypoglycemia, adverse macrovascular events, and inflammation in the Edinburgh type 2 diabetes study. Diabetes Care. 2014;37 (12):3301-3308. doi:10.2337/dc14-0908

16. Schiavon M, Visentin R, Göbel B, et al. Improved postprandial glucose metabolism in type 2 diabetes by the dual glucagon-like peptide-1/glucagon receptor agonist SAR425899 in comparison with liraglutide. Diabetes Obes Metab. 2021;23(8):1795-1805. doi:10.1111/dom. 14394

17. Meier JJ, Rosenstock J, Hincelin-Méry A, et al. Contrasting effects of lixisenatide and liraglutide on postprandial glycemic control, gastric emptying, and safety parameters in patients with type 2 diabetes on optimized insulin glargine with or without metformin: a randomized, open-label trial. Diabetes Care. 2015;38(7):1263-1273. doi:10.2337/ dc14-1984

18. Nagakura J, Yamakawa T, Taguri M, et al. Effects of exenatide and liraglutide on 24-hour glucose fluctuations in type 2 diabetes. Endocr J. 2016;63(3):239-247. doi:10.1507/endocrj.EJ15-0500

19. Bianchi C, Miccoli R, Del Prato S. Hyperglycemia and vascular metabolic memory: truth or fiction? Curr Diab Rep. 2013;13 (3):403-410. doi:10.1007/s11892-013-0371-2

20. Cooper ME, El-Osta A. Epigenetics: mechanisms and implications for diabetic complications. Circ Res. 2010;107(12):1403-1413. doi:10.1161/CIRCRESAHA.110.223552

21. Lu J, Ma X, Zhou J, et al. Association of time in range, as assessed by continuous glucose monitoring, with diabetic retinopathy in type 2 diabetes. Diabetes Care. 2018;41(11):2370-2376. doi:10.2337/dc181131

22. Beck RW, Bergenstal RM, Riddlesworth TD, et al. Validation of time in range as an outcome measure for diabetes clinical trials. Diabetes Care. 2019;42(3):400-405. doi:10.2337/dc18-1444

23. Lu J, Ma X, Shen Y, et al. Time in range is associated with carotid intima-media thickness in type 2 diabetes. Diabetes Technol Ther. 2020;22(2):72-78. doi:10.1089/dia.2019.0251

24. Lanspa MJ, Krinsley JS, Hersh AM, et al. Percentage of time in range 70 to $139 \mathrm{mg} / \mathrm{dL}$ is associated with reduced mortality among critically Ill patients receiving IV insulin infusion. Chest. 2019;156 (5):878-886. doi:10.1016/j.chest.2019.05.016
25. Nauck M, Frid A, Hermansen K, et al. Efficacy and safety comparison of liraglutide, glimepiride, and placebo, all in combination with metformin, in type 2 diabetes: the LEAD (liraglutide effect and action in diabetes)-2 study. Diabetes Care. 2009;32(1):84-90. doi:10.2337/ dc08-1355

26. Zinman B, Gerich J, Buse JB, et al. Efficacy and safety of the human glucagon-like peptide-1 analog liraglutide in combination with metformin and thiazolidinedione in patients with type 2 diabetes (LEAD-4 Met+TZD). Diabetes Care. 2009;32(7):1224-1230. doi: $10.2337 / \mathrm{dc0} 08-2124$

27. Garber A, Henry R, Ratner R, et al. Liraglutide versus glimepiride monotherapy for type 2 diabetes (LEAD-3 Mono): a randomised 52-week, Phase III, double-blind, parallel-treatment trial. Lancet. 2009;373(9662):473-481. doi:10.1016/S0140-6736(08)61246-5

28. Drucker DJ. The role of gut hormones in glucose homeostasis. J Clin Invest. 2007;117(1):24-32. doi:10.1172/JCI30076

29. Campbell JE, Drucker DJ. Pharmacology, physiology, and mechanisms of incretin hormone action. Cell Metab. 2013;17(6):819-837. doi:10.1016/j.cmet.2013.04.008

30. Guo N, Sun J, Chen H, et al. Liraglutide prevents diabetes progression in prediabetic OLETF rats. Endocr J. 2013;60(1):15-28. doi:10.1507/endocrj.EJ12-0094

31. Buteau J. GLP-1 receptor signaling: effects on pancreatic beta-cell proliferation and survival. Diabetes Metab. 2008;34:S73-S77. doi:10.1016/S1262-3636(08)73398-6

32. Chen LN, Lyu J, Yang XF, et al. Liraglutide ameliorates glycometabolism and insulin resistance through the upregulation of GLUT4 in diabetic KKAy mice. Int $J$ Mol Med. 2013;32(4):892-900. doi:10.3892/ijmm.2013.1453

33. Yang J, Ao N, Du J, et al. Protective effect of liraglutide against ER stress in the liver of high-fat diet-induced insulin-resistant rats. Endocrine. 2015;49(1):106-118. doi:10.1007/s12020-014-0480-y

34. Seino Y, Rasmussen MF, Zdravkovic M, et al. Dose-dependent improvement in glycemia with once-daily liraglutide without hypoglycemia or weight gain: a double-blind, randomized, controlled trial in Japanese patients with type 2 diabetes. Diabetes Res Clin Pract. 2008;81(2):161-168. doi:10.1016/j.diabres.2008.03.018

35. Bi Y, Tong GY, Yang HJ, et al. The beneficial effect of metformin on $\beta$-cell function in non-obese Chinese subjects with newly diagnosed type 2 diabetes. Diabetes Metab Res Rev. 2013;29(8):664-672. doi:10.1002/dmrr.2443

\section{Publish your work in this journal}

Diabetes, Metabolic Syndrome and Obesity: Targets and Therapy is an international, peer-reviewed open-access journal committed to the rapid publication of the latest laboratory and clinical findings in the fields of diabetes, metabolic syndrome and obesity research. Original research, review, case reports, hypothesis formation, expert opinion and commentaries are all considered for publication. The manuscript management system is completely online and includes a very quick and fair peer-review system, which is all easy to use. Visit http://www.dovepress.com/testimonials.php to read real quotes from published authors. 